Jahangirnagar University J. Biol. Sci. 5(2): 27-40, 2016 (December)

\title{
Taxonomic checklist of the pteridophytes of Rajkandi Reserve Forest, Moulvibazar, Bangladesh
}

\author{
A. K. M. Kamrul Haque*, Saleh Ahammad Khan, Sarder Nasir Uddin ${ }^{1}$ and \\ Mohammod Abdur Rahim \\ Department of Botany, Jahangirnagar University, Savar, Dhaka-1342, Bangladesh
}

\begin{abstract}
Pteridophytes growing in the Rajkandi Reserve Forest of Moulvibazar district were indentified and a total of 52 species belonging to 30 genera of 20 families have been documented. The family Pteridaceae with nine species was found to be the largest, which was followed by Polypodiaceae with seven, Tectariaceae with six and Thelypteridaceae with five species. The genus Pteris with six species was found as the largest, which was followed by Tectaria with five and Bolbitis, Lygodium and Selaginella, each with 3 species, and the rest of the genera consisted of two or one species. Most of the species were recorded from the woodlands and three species, viz., Tectaria chattagramica and Cyathea gigantea enlisted in Red Data Book of Bangladesh (Khan et al., 2001), were found as common in this reserve forest.
\end{abstract}

Key words: Checklist, Pteridophyte, Reserve forest, Rajkandi, Bangladesh.

\section{INTRODUCTION}

Pteridophytes are an important component of the flora, biodiversity, natural habitats and ecosystems of many areas. A good number of species of this plant group are used as ornamentals and medicinals (Nayar, 1957; Benerjee and Sen, 1980; Dhiman, 1998; Dixit, 1974 \& 1975; Hodge, 1973; Kaushik, 1998; Singh, et al., 1989). Many pteridophytes occurring in Bangladesh are also reported as ethno-medicinally useful (Uddin, et al., 1998; Uddin, et al., 2008; Sarker and Hossain, 2009). However, the pteridophytes of this country have not yet been fully assessed and their status is not yet clear. This plant group has been excluded from most of the floristic studies conducted in Bangladesh and still demand attention for their taxonomic documentation. Prain (1903) was the first who enumerated 98 species of fern from the then East Bengal including the present political boundary of Bangladesh. The report of Prain (1903) did not provide data on the species composition and exact distribution of the pteridophytes in particular areas and forests. In last eleven decades, the nomenclature and taxonomic position of many pteridophytic taxa have also been changed. After the independence of Bangladesh, a very few studies on pteridophytic flora have been done (e.g., Pasha and Mallick, 1980; Pasha and Chakraborty, 1984; Pasha, 1985; Mirza and Rahman, 1997; Uddin and Pasha, 1997; Uddin, 2001; Uddin et al., 2001; Uddin and Hassan, 2012). Recently, Siddiqui et al. (2007) compiled 195 species of pteridophytes from Bangladesh. Most of these works were based mainly on survey of available literatures and examination of deposited voucher specimens at different herbaria. Recently, Hossain et al. (2015) have published a

\footnotetext{
${ }^{1}$ Bangladesh National Herbarium, Chiriakhana Road, Mirpur-1, Dhaka-1216, Bangladesh

* Corresponding author. E-mail: <kamrulhaque1234@gmail.com>. A. K. M. Kamrul Haque
} 
checklist of the pteridophytes of Bangladesh Sunderbans. Information on species composition and diversity of the pteridophytes of most of the areas of this country, especially of the protected areas, are still lacking. Therefore, there is a great scope and need for exploring this plant group in their natural habitats of Bangladesh.

The Rajkandi forest, composed of ca. 2450 hectares of land, is one of the tropical semievergreen forests of this country which falls within the Indo-Burma hot-spot of biodiversity. The Reserve Forest of Rajkandi was declared as a protected area in 1915 by the government of British India. This forest is currently being maintained under the three beats, namely Adampur, Kamarchara and Kurma beats of Rajkandi Forest Range of Sylhet Forest Division (Sayma et al., 2009). The topography of Rajkandi Reserve Forest is undulating with slopes and hillocks that range from 10 to $50 \mathrm{~m}$ in elevation (Rizvi, 1970). The hills are generally low and gently sloping, dissected by numerous valleys, separated by ridges and rising some $50 \mathrm{~m}$ above them (Anon, 2006). These hillocks are scattered and interspersed with numerous streams flowing through the forest that form a scenic topography of Rajkandi Forest. Soil of this forest area ranges from clay loam to pale brown, is acidic ( $\mathrm{pH}$ 5.0) in nature and clay loams in the hills (Anon, 2006). The Rajkandi Reserve Forest seems to harbor a rich flora, species diversity and enormous genetic resources in its diverse habitats and ecosystems, but this forest is not yet explored floristically. Hence, the floristic study of the pteridophytes extant in Rajkandi reserve forests was carried out as the first step in generating the base-line taxonomic information on the pteridophytic flora of this forest for helping the sustainable conservation and management activities there.

\section{MATERIALS AND METHODS}

The Rajkandi Reserve Forest, one of the protected areas of Bangladesh lying between the $24^{\circ} 12^{\prime}-24^{\circ} 17^{\prime} \mathrm{N}$ and $91^{\circ} 51^{\prime}-91^{\circ} 55^{\prime} \mathrm{E}$, is situated in northeast area of Kamalganj upazilla which is located in the southern part of Moulvibazar district. The tropical monsoon prevails in the area with average annual rainfall of $3931 \mathrm{~mm}$ (BBS/UNDP, 2005) and $90 \%$ of the total rainfall occurs in the period between June-September. The average maximum temperature of the study area is $30.7^{\circ} \mathrm{C}$ during May to July, whereas, the average minimum temperature is $30.7^{\circ} \mathrm{C}$ during November to March (BBS/UNDP, 2005). The mean relative humidity is rather high and usually remains between 69-95\% throughout the year (Anonymous, 1986). Soil of Rajkandi forest area ranges from clay loam to pale brown, is acidic ( $\mathrm{pH}$ 5.0) in nature and clay loams in the hills (Anonymous, 2006). 


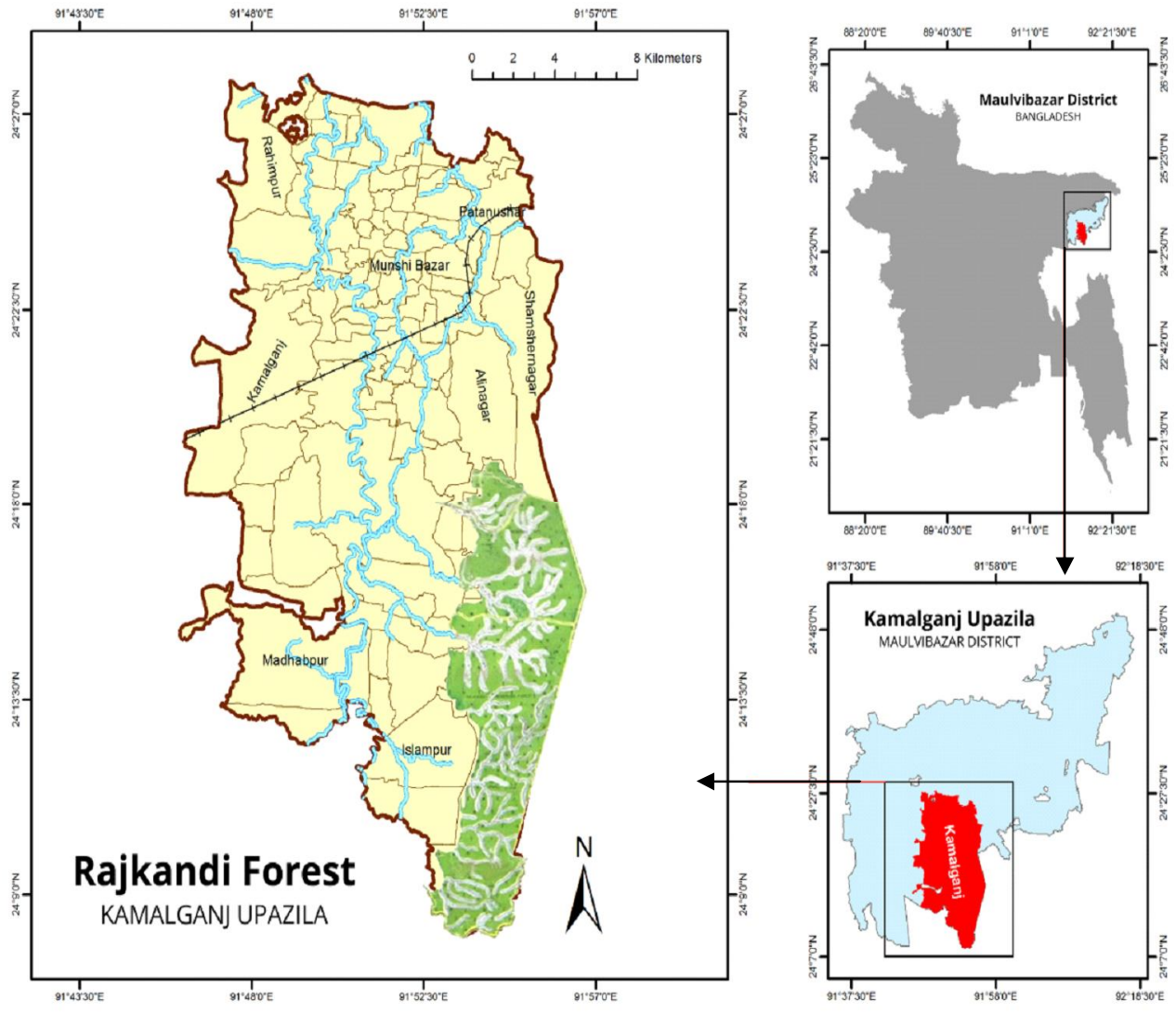

Fig. 1. Map showing the location of Rajkandi forest range in Kamalganj, Moulvibazar, Bangladesh

This study was based on a thorough taxonomic inventory comprised of 25 field trips conducted in different seasons of 2010-2015 throughout the Rajkandi Reserve Forest. Standard herbarium methods and techniques were followed (e.g., Jain and Rao., 1977; Hyland, 1972) in collection, processing, drying and preservation of plant specimens. The representative herbarium specimens were examined at the Bangladesh National Herbarium (DACB), Dhaka University Salar Khan Herbarium (DUSH) and Jahangirnagar University Herbarium (JUH). The specimens were identified by consulting Clarke (1880), Holttum (1968, 1991), Siddiqui et al. (2007) and Smitinand and Larsen (1975; 1989). The relevant type images of pteridophytes available in the web pages of different international herbaria (e.g., K, BM, P) were also matched. The voucher specimens have been preserved at DACB and JUH. Nomenclatural information of each taxon was verified following Flora of China (1995-2013) and the nomenclatural data bases of IPNI (2008) and TROPICOS (2010). The families have been arranged according to the classification system of Pichi (1977). The genera and species under each family have been arranged 
alphabetically. The valid name of each taxon is mentioned with full original citation, followed by the basionym and synonym/s (if available).

\section{RESULTS AND DISCUSSION}

A total of 53 species belonging to 30 genera of 20 families of pteridophytes have been found in Rajkandi Reserve Forest. For each species, up-to-date nomenclature, plant habit, ecology and the examined representative specimens collected from different forest beats of Rajkandi forest range, Kamalganj, Moulvibazar has been mentioned below.

Family: EQUISETACEAE Michx. ex DC. Essai Propr. Méd. Pl. 49. (1804).

Equisetum L. 2: 1061. Sp. Pl.(1753).

Equisetum diffusum D. Don. Prodr. Fl. Nepal. 19. (1825). Equisetum mekongense C.N. Page.(1974).

Terrestrial, erect herbs, creeping rhizome, fibrous root in nodes. In edge of the forest; on semi dry soil. In Bangladesh, it occurs in Rajshahi, Sylhet, Mymensingh,Chittagong and Rangamati.

Representative specimens: Adampur, 29 Jun 2015, 2276, Kamrul 2277 (JUH). Kamarchara, 29 Jun 2015, Kamrul 2261 (JUH). Kurma, 30 Jun 2015, Kamrul 2278 (JUH).

Family: LYCOPODIACEAE P. Beauv. ex Mirb. Hist. Nat. Vég. 4: 293 (1802).

Palhinhaea Franco \& Vasc. Bol. Soc. Brot., sér. 2, 41: 24. (1967).

Palhinhaea cernua (L.) Vasc. \& Franco. Bol. Soc. Brot., sér. 2, 41: 25 (1967). Lycopodium cernuum L.Sp. Pl. 2: 1103 (1753). Lepidotis cernua (L.) P. Beauv (1805).

Erect terrestrial herb, roots in clumps. At forest margins and shaded places; on moist soil. In Bangladesh it occurs in Chittagang and Sylhet.

Representative specimens: Adampur, 4 Jun 2014, Kamrul 1958 (JUH). Kamarchara, 4 Jun 2014, Kamrul 1960 (JUH). Kurma, 5 Jun 2014, Kamrul 1961 (JUH).

Family: SELAGINELLACEAE Willk Anleit. Stud. Bot. 2: 163 (1854).

Selaginella P. Beauv. Prodr. Aetheogam. 101 (1805).

Selaginella ciliaris Bull. Acad. Roy. Sci. Bruxelles, 10 (1): 231, no. 136 (1843). Lycopodium ciliare Retz. (1789).

Terrestrial creeping herb, rooting at intervals. On stream bank; on moist soil. In Bangladesh it occurs northern and eastern part of the country.

Representative specimens: Adampur, 7 Jun 2014, Kamrul 1999 (JUH). Kamarchara, 7 Jun 2014, Kamrul 2000 (JUH). Kurma, 8 Jun 2014, Kamrul 2001 (JUH).

Selaginella semicordata (Wall.) Spring. Fl. Bras. (2): 122 (1840). Lycopodium semicordatum Wall ex. Hook. \& Grev. (1831).

Terrestrial, erect herb, rooting throughout. Shady slope of hills;on semi moist soil. In Bangladesh it occurs eastern part of the country.

Representative specimens: Adampur, 7 Jun 2014, Kamrul 1999 (JUH). Kamarchara, 7 Jun 2014, Kamrul 2000 (JUH). Kurma, 8 Jun 2014, Kamrul 2001 (JUH); 7 Jun 2014, M. A. Rahim 2508 (JUH).

Selaginella tenuifolia Spring, Mém. Acad. Sci. Belg. 24: 253 (1850). 
Terrestrial erect herb, rhizome prominant. Shady slope of hills; on semi moist soil. In Bangladesh it occurs it occurs eastern part of the country.

Representative specimens: Adampur, 7 Jun 2014, Kamrul 1997 (JUH).

Family: HELMINTHOSTACHYACEAE Ching. Bull. Fan Mem. Inst. Biol.Bot.10: 235 (1941).

Helminthostachys Kaulf. Enum. Filic. 28 (1824).

Helminthostachys zeylanica (L.) Hook. Gen. Fil., pl. 47, B. (1842). Osmunda zeylanica L. (1753). Botrychium zeylanicum (L.) Sw. (1801).

Terrestrial herb, rhizome fleshy. In forests edges; on moist soil. In Bangladesh it occurs in Chadpur, Chittagong, Mymensingh, Tangail, Shylleht, Hill tracts and Sunderban.

Representative specimens: Adampur, 4 Jun 2014, Kamrul 2025 (JUH); M.A. Rahim 2490 (JUH).

Family: OPHIOGLOSSACEAE Martinov. Tekhno-Bot. Slovar. 438 (1820).

1. Ophioglossum L., SP. PL. 2: 1062 (1753).

Ophioglossum reticulatum L., Sp. Pl. 2: 1063 (1753). Ophioglossum cordifolium Roxb. (1844).

Terrestrial, rhizomatous, erect herbs. In edge of the grass land; on moist soil. In Bangladesh it occurs in Dhaka and Mymensingh districts

Representative specimens: Adampur, 29 Jun 2015, Kamrul 2267 (JUH). Kamarchara, 29 Jun 2015, Kamrul 2268 (JUH). Kurma, 30 Jun 2015, Kamrul 2269 (JUH).

Family: MARATTIACEAE Kaulf. Enum. Filic31. (1824).!! = nom. Cons.

1. Angiopteris Hoffm. 12 (Cl. Phys.): 29 (1796).

Angiopteris evecta (G. Forst.) Hoffm. Commentat. Soc. Regiae Sci. Gott. 12: 29, t. 5. (1794). Polypodium evectum G. Forst. (1786). Angiopteris acrocarpa de Vriese(1853).

Terrestrial herb, rhizomes erect. In forests, valleys, scrubjungle; on moist soil. In Bangladesh it occurs in Chittagong, Cox's Bazar and Sylhet district

Representative specimen: Adampur, 6 Feb 2014, Kamrul 1916 (JUH); M.A. Rahim 2377 (JUH).

Angiopteris sylhetensis de Vriese (1853).

Terrestrial herb, rhizomes erect. In forests, valleys, scrubjungle; on moist soil. In Bangladesh, it occurs in Sylhet.

Representative specimens: Adampur, 6 Feb 2014, Kamrul 1917 (JUH); M.A. Rahim 2378

(JUH). Kamarchara, 6 Feb 2014, Kamrul 1918 (JUH). Kurma, 7 Feb 2014, Kamrul 1919 (JUH.

Family: SALVINIACEAE Martinov, Tekhno-Bot. Slovar. 559 (1820).

1. Salvinia Ség. Fl. Veron.3: 52 (1754).

Salvinia cucullata Roxb. Calcutta J. Nat. Hist. 4: 470 (1844).

Free floating, rhizomatous herbs. In stagnant water. In Bangladesh it occurs throughout the country.

Representative specimen: Kurma, 30 Jun 2015, Kamrul 2263, 2264 (JUH); M.A. Rahim 2601 (JUH).

Salvinia natans (L.) All. Fl. Pedem.2: 289 (1785). Marsilea natans L. (1753).

Free floating, rhizomatous herbs. In stagnant water. In Bangladesh it occurs throughout the country. 
Representative specimens examined: Kurma, 30 Jun 2015, Kamrul 2264, 2265 (JUH); M.A. Rahim 2602 (JUH).

Family: MARSILEACEAE Mirb. Hist. Nat. Vég. 5:126. (1802).

1. Marsilea L. Sp. Pl. 2: 1099. (1753).

Marsilea minuta L. Mant. Pl. Alt. [2]: 308 (1771). Marselia aegyptica wall. (1829).

Terrestrial herb, rhizomes creeping. In rice fields, ditches. In Bangladesh it occurs in cox's Bazar, Dhaka, Rajshahi, Rangpur and Manikganj.

Representative specimen: Adampur, 30 Jun 2015, Kamrul 2261 (JUH).

Marsilea quadrifolia L. Sp. Pl. 2: 1099-1100 (1753). Marselia europa Desv. (1827).

Terrestrial herb, rhizomes creeping. In rice fields; on moist soil. In Bangladesh it occurs throughout the country.

Representative specimen: Adampur, 30 Jun 2015, Kamrul 2260 (JUH); M.A. Rahim, 2603 (JUH).

Family: LYGODIACEAE M. Roem., Handb. Allg. Bot. 3: 520 (1840).

Lygodium Sw., J. Bot. (Schrader). 1800 (2): 7, 106 (1801).

Lygodium circinatum (Burm. f.) Sw. Syn. Fil. 153 (1806). Ophioglossum circinnatum Burm. f. (1768).

Terrestrial herb, climbing, rhizome creeping. In shaded places in forests; on moist soil. In Bangladesh it occurs in Chittagong.

Representative specimens: Adampur, 4 Jun 2014, Kamrul 1957 (JUH); M.A. Rahim 2479 (JUH).

Lygodium flexuosum (L.) Sw., J. Bot.(Schrader). 1800 (2): 7, 106 (1801). Ophioglossum flexuosum L. (1753). Lygodium pilosum Desv. (1827).

Terestrial climbing herbs, rhizome creeping. In scrub jungle; on moist soil. In Bangladesh it occurs throughout the country.

Representative specimens: Adampur, 4 Jun 2014, Kamrul 1953 (JUH). Kamarchara, 4 Jun 2014, Kamrul 1954 (JUH). Kurma, 5 Jun 2014, Kamrul 1955 (JUH); M.A. Rahim 2480 (JUH).

Lygodium giganteum Tagawa \& K. Iwats. Acta Phytotax. Geobot. 22: 97, f. 1 (1967).

Terrestrial, climbing herb, rhizome creeping. In slopes; on moist soil. In Bangladesh, it occurs in Chittagong.

Representative specimens: Adampur, 4 Jun 2014, Kamrul 1951 (JUH). Kurma, 5 Jun 2014, Kamrul 1952 (JUH).

Family: PTERIDACEAE E.D.M. Kirchn., Schul-Bot. 109 (1831).

Aleuritopteris Fée. Mem. Foug., Gen. Filic. 5: 153-154 (1850-1852).

Aleuritopteris farinosa (Forssk.) Fée.Mem. Foug., Gen. Filic. 153, t. 12B. (1850-1852). Cheilanthes farinosa (Forssk.) Kaulf. (1824).

Terrestrial herb, rhizome short. In shaded forest; on moist soil. In Bangladesh it occurs in Sylhet.

Representative specimen: Adampur, 6 Feb 2014, Kamrul 1935 (JUH).

Onychium Kaulf. Berlin. Jahrb. Pharm. Verbundenen Wiss. 21: 45 (1820).

Onychium siliculosum (Desv.) C. Chr. Index Filic. 8: 469 (1906). Pteris siliculosa Desv. (1811). Acrostichum viviparum L. f. (1782).

Terrestrial herb, rhizomes ascending. In forest valleys; on moist soil. In Bangladesh it occurs in Chittagong Hill tract. 
Representative specimen: Adampur, 4 Jun 2014, Kamrul 1971 (JUH); M.A. Rahim 2481

(JUH).

Pityrogramma (L) Link. Handbuch. 3: 19-20 ( 1833).

Pityrogramma calomelanos (L.) Link. Handbuch 3: 20 (1833). Acrostichum

calomelanos L. (1753). Neurogramma calomelanos (L.) Diels. (1899).

Terrestrial herb, rhizome erect. In shded forest; on moist soil. In Bangladesh it occurs in Chittagong, Maulvibazar and Panchagarh.

Representative specimens: Adampur, 4 Jun 2014, Kamrul 1988 (JUH). Kurma, 5 Jun 2014, Kamrul 1989 (JUH).

Pteris L.Sp. Pl. 2: 1073 (1753.)

Pteris ensiformis Burm. f. Fl. Indica 230 (1768).

Terrestrial herb, rhizome ascending. Inside forests, streamsides; on moist soil. In Bangladesh it is found in forest areas.

Representative specimen: Adampur, 4 Jun 2014, Kamrul 1985 (JUH); M.A. Rahim 2482(JUH).

Pteris griffithii Hook.; Sp. Fil. 2: 170. t. 123A (1858).

Terrestrial herb, rhizome erect. Shady forest floor; on moist soil. In Bangladesh, it occurs in Sylhet.

Representative specimen: Adampur, 4 Jun 2014, Kamrul 1976 (JUH).

Pteris pseudopellucida Ching, Lingnan Sci. J., 15 (3): 395 (1936).

Terrestrial herb, rhizome erect. In hilly forest; on semi moist soil. In Bangladesh, it occurs in Sylhet.

Representative specimens: Adampur, 7 Apr 2014, Kamrul 1940 (JUH). Kamarchara, 7 Apr 2014, Kamrul 1941 (JUH). Kurma, 8 Apr 2014, Kamrul 1942 (JUH).

Pteris semipinnata L. Sp. Pl. 2: 1076 (1753).

Terrestrial herb, rhizomes erect. Scrub jungle, on semi dry soil. In Bangladesh it occurs in Chittagong, Sylhet.

Representative specimens: Adampur, 7 Jun 2014, Kamrul 1979 (JUH); Kurma, 8 Jun 2014, Kamrul 1980 (JUH).

Pteris venusta Kuntze, Bot. Zeitung (Berlin), 6: 195-196 (1848). Pteris matsudai Masam. (1935).

Terrestrial herb, rhizome erect. In open forests; on semi dry soil. In Bangladesh it occurs in Chittagong, Sylhet.

Representative specimens: Adampur, 8 Jun 2014, Kamrul 1977 \& 1978 (JUH); M.A. Rahim 2487(JUH).

Pteris vittata L., Sp. Pl. 2: 1074 (1753). Pteris longifolia L (1753).

Terestrial, rhizomatous, prostrate herbs. In marginal land; on semi dry soil. In Bangladesh it occurs throughout the country.

Representative specimen: Adampur, 7 Jun 2014, Kamrul 1975 (JUH); M.A. Rahim 2485 (JUH).

Family: CYATHEACEAE Kaulf. Wesen Farrenkr. 119 (1827).

Cyathea Sm. Mém. Acad. Roy. Sci. (Turin)5: 416 (1793).

Cyathea gigantea (Wall. ex Hook.) Holttum, Gard. Bull. Straits Settlem. 8(4): 318 (1935). Alsophila gigantea Wall. ex Hook. (1844). 
Terrestrial small tree like. Beside shady hilly streams; on moist soil. In Bangladesh it occurs in Sylhet, Cox's Bazar and chittagong

Representative specimens: Adampur, 4 Jun 2014, Kamrul 1937 (JUH). Kamarchara, 4 Jun 2014, Kamrul 1938 (JUH). Kurma, 5 Jun 2014, Kamrul 1939 (JUH); M.A. Rahim 2453(JUH).

Family: DENNSTAEDTIACEAE Lotsy. Vortr. Bot. Stammesgesch. 2: 655 (1909).

Microlepia C. Presl. Tent. Pterid. 124-125, pl. 4, f. 21-23 (1836).

Microlepia strigosa (Thunb.) C. Presl. Abh. Königl. Böhm. Ges. Wiss. ser. 56: 455 (1851). Trichomanes strigosum Thunb. (1784). Microlepia formosana Ching. (1959).

Terrestrial herb,creeping with long rhizome. Inside forest; on semi dry soil. In Bangladesh, it occurs in Chittagong hilltracts.

Representative specimen: Adampur, 4 Jun 2014, Kamrul 1964 (JUH); M.A. Rahim 2454 (JUH).

Family: LINDSAEACEAE C. Presl ex M.R. Schomb. Reis. Br.-Guiana 3: 883, 1048 (1848).

Lindsaea Dryand. ex Sm. Mém. Acad. Roy. Sci. (Turin) 5: 413, pl. 9, f. 4 (1793).

Lindsaea ensifolia Sw. J. Bot. (Schrader) 1800(2): 77 (1801). Adiantum ensifolium (SW.) Poir. (1810).

Terrestrial, rhizomes creeping. In scrub jungle; on moist soil. In Bangladesh it occurs throughout the country.

Representative specimens: Adampur, 4 Jun 2014, Kamrul 1962 (JUH). Kamarchara, 4 Jun 2014, Kamrul 1963 (JUH). Kurma, 5 Jun 2014, Kamrul 1964(JUH).

Family: POLYPODIACEAE J. Presl \& C. Presl, Delic. Prag. 159 (1822).

Drynaria (Bory) J. Sm., J. Bot. (Hooker) 4: 60 (1842).

Drynaria quercifolia (L.) J. Sm., J. Bot. (Hooker). 3: 398 (1841). Polypodium quercifolium L. (1753).

Epiphytic herbs, rhizomatous. Inside forest; on trees. In Bangladesh it occurs throughout the country.

Representative specimen: Adampur, 4 Jun 2014, Kamrul 1944 (JUH); M.A. Rahim 2455 (JUH).

Drynaria sparsisora (Desv.) T. Moore, Index Fil., 348. (1862). Polypodium sparsisorum Desv. (1811). Drynaria linnei Bory ex Bedd. (1869).

Epiphytic, rhizomatous. Inside forest, river bank; on trees. In Bangladesh it occurs in Dhaka.

Representative specimen: Adampur, 4 Jun 2014, Kamrul 1943 (JUH).

Leptochilus Kaulf. Enum. Filic. 147, pl. 1, f. 10 (1824).

Leptochilus pedunculatus (Hooker \& Greville) Fraser-Jenkins, Taxon. Revis. Indian Subcontinental Pteridophytes. 62. (2008). Ceterach pedunculatum Hook. \& Grev. (1827). Colysis pedunculata (Hook. \& Grev.) Ching. (1933).

Terrestrial herb with creeping rhizome. In shaded forest; on moist soil. In Bangladesh it occurs in Sylhet.

Representative specimen: Adampur, 4 Jun 2014, Kamrul 1933 (JUH).

Microsorum Link, Hort. Berol. 2: 110 (1833).

Microsorum punctatum (L.) Copel., Univ. Calif. Publ. Bot. 16: 111 (1929). Acrostichum punctatum L. (1763). Phymatodes lingulata (Sw.) C. Presl (1836). 
Epiphytic herbs. Inside forest, river bank; on trees. In Bangladesh it occurs throughout the country.

Representative specimens: Adampur, 4 Jun 2014, Kamrul 1965 (JUH). Kamarchara, 5 Jun 2014, Kamrul 1966 (JUH). Kurma, 6 Jun 2014, Kamrul 1967 (JUH); M.A. Rahim 2456 (JUH).

Neocheiropteris Christ . Bull. Soc. Bot. France: Mem.1: 21 (1905).

Neocheiropteris ovatum (Fée) Fraser-Jenk. New Sp. Syndr. Indian Pteridol. 180 (1997). -Polypodium ovatum. Wall. ex Hook. et Grev. (1827).

Terrestrial herb, with creeping rhizome. In shaded forest; on moist soil. In Bangladesh it occurs in Sylhet.

Representative specimen: Adampur, 7 Jun 2014, Kamrul 1970 (JUH).

Pyrrosia Mirb., Hist. Nat. Vég. 3. 471; 5: 91 (1802).

Pyrrosia lanceolata (L.) Farw. Amer. Midl. Naturalist 12(8): 245 (1931). Acrostichum lanceolatum L. (1753).

Herb, epiphytic, rhizome slender. In shaded forest; on moist soil. In Bangladesh it occurs in Chittagong, Dhaka and Sylhet.

Representative specimens: Adampur, 3 Jun 2014, Kamrul 1986 (JUH). Kurma, 4 Jun 2014, Kamrul1987 (JUH).

Pyrrosia nuda (Giesenh.) Ching. Bull. Chin. Bot. Soc.1(1): 70 (1935). Cyclophorus nudus (Giesenh.) C. Chr. (1905).

Epiphytic herb, rhizome slender. On tree trunks. In Bangladesh it occurs throughout the country.

Representative specimens: Adampur, 4 Jun 2014, Kamrul 1947 (JUH). Kurma, 5 Jun 2014, Kamrul 1948 (JUH); M.A. Rahim 2457 (JUH).

Family: BLECHNACEAE Newman. Hist. Brit. Ferns (ed. 2) 8 (1844).

Blechnum L. Sp. Pl. 2: 1077 (1753).

Blechnum orientale L. Sp. Pl.2: 1077. (1753). Blume (1828). Blechnopsis orientalis (L.) C. Presl. (1851).

Terrestrial herb, rhizome erect. Open places of forest; on moist soil. In Bangladesh it occurs throughout the country.

Representative specimens: Adampur, 3 Jun 2014, Kamrul 1931 (JUH); M.A. Rahim 2423(JUH). Kurma, 4 Jun 2014, Kamrul 1932 (JUH).

Family: THELYPTERIDACEAE Ching ex Pic. Serm., Webbia 24: 709 (1970).

Ampelopteris Kunze, Bot. Zeitung (Berlin) 6: 114-115 (1848).

Ampelopteris prolifera (Retz.) Copel., Gen. Fil. 144 (1947). Hemionitis prolifera Retz. (1791). Polypodium proliferum (Retz.) Hook (1864).

Terrestrial herb, trailing, rhizome creeping. In marginal land; on moist soil. In Bangladesh, it occurs in Panchagarh, Sylhet

Representative specimens: Adampur, 29 Jun 2015, Kamrul 2271 (JUH). Kamarchara, 29

Jun 2015, Kamru 2272 (JUH). Kurma, 30 Jun 2015, Kamrul 2273 (JUH).

Cyclosorus Link, Hort. Berol. 2: 128 (1833).

Cyclosorus crinipes (Hook.) Ching, Bull. Fan Mem. Inst. Biol., Bot. 8: 179 (1938).

Nephrodium crinipes Hook. (1962). Christella crinipes (Hook.) Holttum (1974).

Terrestrial herb, decumbent, rhizome erect. In marginal land; on moist soil. In Bangladesh, it occurs in Chittagong and Cox's Bazar districts 
Representative specimen: Adampur, 7 Apr 2014, Kamrul 1934 (JUH).

Cyclosorus dentatus (Forssk.) Ching, Bull. Fan Mem. Inst. Biol., Bot. 8: 206 (1938). Polypodium dentatum Forssk. (1775). Christella dentata (Forssk.) Brownsey \& Jermy (1973).

Terrestrial herb, decumbent, rhizome creeping. In open scrub; on moist soil. In Bangladesh, it occurs in Chittagong and Dhaka.

Representative specimens: Adampur, 29 Jun 2015, Kamrul 2273 (JUH). Kamarchara, 29 Jun 2015, Kamrul 2274 (JUH). Kurma, 30 Jun 2015, Kamrul 2275 (JUH).

Pronephrium C. Presl. Abh. Königl. Böhm. Ges. Wiss. ser. 5 6: 618-619 (1851).

Pronephrium nudatum(Roxb. ex Griff.) Holt. Blumea 20(1): 111 (1972). Polypodium nudatum Roxb. (1844). Nephrodium moulmeinense Bedd. (1876).

Terrestrial herb, rhizome stout creeping. Scrub jungle, stream sides; on semi dry soil. In Bangladesh it occurs in Mymensingh, Sylhet district.

Representative specimens: Adampur, 7 Jun 2014, Kamrul 1990 (JUH). Kamarchara, 7 Jun 2014, Kamrul 1991 (JUH). Kurma, 8 Jun 2014, Kamrul 1992 (JUH); M.A. Rahim 2518(JUH).

Pronephrium triphyllum (Sw.) Holttum Blumea 20 (1): 12 (1972). Meniscium triphyllum Sw. (1801). Cyclosorus triphyllus (Sw.) Tardieu (1938).

Terrestrial herb with long creeping rhizomes. Inside forests; on moist soil. In Bangladesh, it occurs in Sylhet.

Representative specimen: Adampur, 7 Jun 2014, Kamrul 1995 (JUH).

Family: ASPLENIACEAE Newman, Hist. Brit. Ferns. 6 (1840).

Asplenium L, Sp. Pl. 2: 1078 ( 1753).

Asplenium unilaterale Lam. Encycl. 2 (1): 305 (1786). Asplenium szechuanense Ching. (1965).

Terrestrial herb, rhizome creeping. In shaded stream banks; on moist soil. In Bangladesh it occurs in Chittagong.

Representative specimens: Adampur, 3 Jun 2014, Kamrul 1920 (JUH). Kamarchara, 3 Jun 2014 Kamrul 1921 (JUH). Kurma, 4 Jun 2014, Kamrul 1922 (JUH).

Family: DRYOPTERIDACEAE Herter. Revista Sudamer. Bot. 9: 15 (1949). !! = nom.

Cons.

Bolbitis Schott. Gen. Fil. pl. 14 (1834).

Bolbitis appendiculata (Willd.) K. Iwats. Acta Phytotax. Geobot.18(2-3): 48-49 (1959). Acrostichum appendiculatum Willd. (1810). Egenolfia appendiculata (Willd.) J. Sm.(1866).

Terrestrial herb, rhizome creeping. In forest; on semi dry soil.

Representative specimen: Adampur, 4 Jun 2014, Kamrul 1928 (JUH); M.A. Rahim 24758 (JUH).

Bolbitis costata Ching. Index Filic., Suppl. 3, 47 (1934). Campium costatum C. Pres1 (1836). Gymnopteris costata (C.Presl) Bedd. (1876).

Terrestrial herb, rhizomatous. Hilly forests near streams; on moist soil. In Bangladesh, it occurs in Chittagong.

Representative specimens: Adampur, 7 Apr 2014, Kamrul 1925 (JUH). Kamarchara, 7 Apr 2014, Kamrul 1929 (JUH). Kurma, 8 Apr 2014, Kamrul 1930 (JUH). 
Bolbitis heteroclita (C. Presl) Ching. Index Filic. Suppl. 3: 48 (1934). Acrostichum heteroclitum C. Presl. (1825). Bolbitis annamensis Tardieu \& C. Chr. (1938).

Terrestrial herb, rhizome creeping. Creeping on rocks trees in forest near streams; on moist soil. In Bangladesh it occurs in Chittagong.

Representative specimen: Adampur, 7 Apr 2014, Kamrul 1927 (JUH).

Family: ATHYRIACEAE Alston, Taxon 5: 25 (1956).

Diplazium Sw., J. Bot. (Schrader). 1800 (2): 61 (1801).

Diplazium esculentum (Retz.) Sw., J. Bot. (Schrader).1801(2): 312 (1803). Hemionitis esculenta Retz. (1791). Athyrium esculentum (Retz.) Copel. (1908).

Terrestrial herb, rhizome erect. Hilly forests near streams; on moist soil. In Bangladesh, it occurs in Chittagong, Cox's Bazaar, Rajshahi, Rangpur and Sylhet.

Representative specimens: Adampur, 21 Aug 2011, Kamrul 1945 (JUH); M.A. Rahim 2145 (JUH). Kamarchara, 21 August 2011, Kamrul 1946 (JUH).

Family: TECTARIACEAE Panigrahi. J. Orissa Bot. Soc. 8: 41 (1986).

Pleocnemia C. Presl. Tent. Pterid. 183, pl. 7, f. 12 (1836).

Pleocnemia irregularis (Presl.) Holttum. Kew Bull., 29: 347 (1974). Polypodium irregulare C. Presl. (1825).

Terrestrial herb, rhizome creeping. Hilly forests; on moist soil. In Bangladesh, it occurs in Cox's Bazar.

Representative specimen: Adampur, 7 Jun 2014, Kamrul 1974 (JUH).

Tectaria Cav. Anales Hist. Nat. 1(2): 115. (1799).

Tectaria chattagramica (Clarke) Ching; Sinensia 2 (2) 35 (1931).

Polypodiumchattagramica Clarke (1880).

Terrestrial herb, rhizomatous. Hilly forests; on moist soil. In Bangladesh, it occurs in Chittagong district.

Representative specimens: Adampur, 7 Jun 2014 Kamrul 2006 (JUH); M.A. Rahim 2512 (JUH). Kurma, 8 Jun 2014 Kamrul 2007 (JUH).

Tectaria heterosora (Bak.) Ching, Senensia 2 (2): 35 (1931). Nephrodium heterosorum Bak. (1874).

Terrestrial, rhizome widely creeping. Shady valleys; on moist soil. In Bangladesh, it occurs in Chittagong and Cox's Bazar .

Representative specimen: Adampur, 7 Jun 2014, Kamrul 2014 (JUH); M.A. Rahim 2513 (JUH). Kamarchara, 7 Jun 2014, Kamrul 2015 (JUH). Kurma, 8 Jun 2014, Kamrul 2016 (JUH), M.A. Rahim 2516 (JUH).

Tectaria paradoxa (Fée) Sledge, Kew Bull., 27 (3): 413-415 (1972). Aspidium paradoxum Fée. (1852).

Terrestrial herb, rhizome erect. Forest floor; on moist soil. In Bangladesh, it occurs in Chittagong district.

Representative specimen: Adampur, 7 Jun 2014, Kamrul 2017 (JUH); M.A. Rahim 2514 (JUH).

Tectaria polymorpha (Wall. ex Hook.) Copel. Philipp. J. Sci. 2 (6): 413 (1907). Aspidium polymorphum Wall. ex Hook. (1862). Aspidium nantoense Hayata.(1919).

Terrestrial herb, rhizome sub-erect. Forest floor; on moist soil. In Bangladesh it occurs in Chittagong.

Representative specimen: Adampur, 7 Jun 2014, Kamrul 2013, 2014 (JUH). 
Tectaria vasta (Blume) Copel. Philip J. Sci. 2 c.: 411 (1907). Nephrodium vastum Bak. (1867). Aspidium alatum Hook. \& Grev. (1831).

Terrestrial herb, rhizome erect. Hilly shady places, near streams; on semi dry soil. In Bangladesh it occurs in Chittagong.

Representative specimens: Adampur, 30 Jun 2015, Kamrul 2260 (JUH). Kurma, 30 Jun 2015, Kamrul 2261 (JUH); M.A. Rahim 2517(JUH).

Among the total 52 pteridophyte species recorded from Rajkandi Resrve Forest, 50 were found in Adampur beat, which was followed by 27 and 18 species, respectively in Kurma and Kamarchara beat and 17 species were commonly found in these three beats. These data indicate that the Adampur beat harbors most of the pteridophytes of Rajkandi forest range. A total of 46 species were found as terrestrial. Only five species were epiphytic and two species were aquatic. The family Pteridaceae with nine species, Polipodiaceae with seven, Tectariaceae with six and Thelypteridaceae with five species constitute $51.92 \%$ of total fern flora of the area, whereas, remaining sixteen families constitute $48.08 \%$. Pteris with six species was found as the largest genus, which was followed by Tectaria with five and Bolbitis, Lygodium and Selginella, each with 3 species. The rest of the genera were consisted of two or one species each. Uddin and Pasha (1997) recorded 16 fern allies, Mirza and Rahman (1997) listed 161 species of fern and fern allies and Siddiqui et al. (2007) compiled 195 species of pteridophytes from Bangladesh. Considering Siddiqui et al. (2007), the Rajkandi Reserve Forest harbors 26.66 percent of the pteridophyte species of Bangladesh and hence, it can be concluded that this reserve forest is an important area for harboring the pteridophytes of this country. In respect to the size of land area, the species composition of the pteridohytes of Rajkandi Reserve Forest seems higher than that of few other protected areas of Bangladesh. For example, 41 fern species were recorded from Rampahar and Sitapahar (Uddin and Hassan, 2012) and 24 species from Sundarbans (Rahman et al., 2016). Two species of pteridophytes occurring in Rajkandi Reserve Forest, viz., Cyathea gigantea, and Tectaria chattagramica, have been enlisted as LR (cd)- (Lower risk, conservation dependent) and DD (Data deficient) respectively in Red Data Book by Khan et al. (2001). But these been commonly found in this reserve forest with normal natural regeneration.

Throughout the forests of Rajkandi, woodlands were found as the most common habitats that harbored a total of 25 species, which was followed by stream bank harboring 13 species, the scrub jungles nine species, grass land five species, valley four species, marginal lands three species and aquatic habitat two species. It indicates that the pteridophytic species of the study area were variable in preferring their habitats. In Rajkandi Reserve Forests, most of the natural habitats were found to be disturbed in different extents, mainly due to various anthropogenic activities and weak forest management. As the pteridophytes are very susceptible to habitat disturbances, therefore, their natural habitats should be conserved through the effective and sustainable conservation programs.

Acknowledgements: Authors are thankful to the authority of the Bangladesh Forest Department for their co-operation during the field works. They are grateful to the 
authorities of the following herbaria for allowing consulting their libraries and using their herbarium materials, viz. Bangladesh National Herbarium (DACB) and Dhaka University Salar Khan Herbarium.

\section{REFERENCES}

Akhter, S., Alamgir, M., Sohel, M.S.I. and Rana, M.P. 2009. Assesment of Cultivated NTFPs Species Stock in Sylhet forest Division: A Case Study from North Sylhet Range 1 and Rajkandi Range, Bangladesh. Int. J, for Usuf. Mngt. 10 (2): 11-19.

Anonymous. 2006. Status, Land Bases and Management Practices of Protected Areas in Bangladesh: An overview. B.Sc. (Hons.). Review Paper No. 24. Dept. of Forestry, Shahjalal Universty of Science and Technology, Sylhet, Bangladesh. pp. 7-72.

Anonymous. 1986. Sylhet Forest Division, A mimiographical report on various aspects of Sylhet Forest Division, 54 pp.

Benerjee, R.D. and Sen, S.P. 1980. Antibiotic activities of Pteridophytes. Ec. Bot. 34(2): 284-298.

BBS/UNDP - Bangladesh Bureau of statistics, 2005. Compendium of Environment Statistics of Bangladesh. Ministry of Planning, Government of the Peoples Republic of Bangladesh. pp. 12-227.

Clarke, C.B. 1880. A Review of the Ferns of Northern India. Indian reprint 1973, Trans. Linn. Soc.London-II. Bot. 1: 425-611.

Dhiman, A.K. 1998. Ethnomedicinal uses of some Pteridophytic species in India. Indian Fern. J. 15: $61-65$.

Dixit, R.D. 1974. Ferns - a much neglected group of medicinal plants. I. J. Res. Indian Med. 9(4): 59-68.

Dixit, R.D. 1975. Ferns - a much neglected group of medicinal plants. III. J. Res. Indian Med. 10(2): 74-90.

Holttum, R.E. 1968. A Revised Flora of Malaya. Fern of Malaya 2. Singapore Govt. Printing Office,Singapore. pp. 1-653.

Holttum, R.E. 1991. Flora Malesiana. Pteridophyta. Fern and Fern allies. ser. 2, vol. 2 , part 1. Foundation Flora Malesiana. pp.1-132.

Hossain, G.M., Rahman, M.S., Khan, S.A. and Uddin, S.N. 2015. A floristic and ecological study on the Pteridophytes of Sundarbans mangrove forest of Bangladesh. Bull. Bangladesh National Herb. 4: 51-66.

Hodge, W.H. 1973. Fern food of Japan and the problem of toxicity. Amer. Fern. J. 63: 77-80.

Hyland, B.P.M. 1972. A technique for collecting botanical specimens in rain forest. Flora Malesiana Bulletin 26: 2038-2040.

IPNI. 2008. The International Plant Names Index. Published on the Internet. http://www.ipni.org [accessed 2010].

Kaushik, P. 1998. Ethnobotanical Importance of Ferns of Rajasthan: Indigenous Medicinal Plants. Today and Tomorrow Printers and Publication, New Delhi. pp. 61-66.

Khan, M.S., Rahman, M.M. and Ali, M.A. (eds) 2001. Red Data Book of Vascular Plants of Bangladesh. Bangladesh National Herbarium, Dhaka, Bangladesh. pp. 1-179.

Mirza, M.M. and Rahman, M.M. 1997. An annotated checklist of ferns and fern allies of Bangladesh. Bangladesh J. Plant Taxon. 4 (2): 47-69.

Nayar, B.K. 1957. Medicinal ferns of India. Bull. Nat. Bot. Gdn. 58: 1-38.

Pichi, S.R.E.G. 1977. Tentamen pteridophytorum genera in taxonomicum ordinem redigendi. Webbia 31: 313-512.

Prain, D. 1903. Bengal Plants, vol. 2. Indian reprint 1981. Bishen Singh Mahendra Pal Singh, Dehra Dun, India. pp. 663-1319. 
Pasha, M.K. and Mallik, A.U. 1980. Ferns of Bangladesh-I. Chittagong Univ. Stud. Pt. II. (Sci.) 2(4):13-26.

Pasha, M.K. and Chakraborty, R. 1984. Ferns of Bangladesh-III. The genus Adiantum. Chittagong Univ. Stud. Pt. II. (Sci.) 8(2): 113-121.

Pasha, M. K. 1985. A systematic account of Selaginella from Bangladesh. Bangladesh J. Bot. 14(2): 97-107.

Rahman, M.S., Hossain, M.G., Khan, S.A. and Uddin, S.N. 2016. An Annotated Checklist Of The Vascular Plants of Sundarban Mangrove Forest of Bangladesh. Bangladesh J. Plant Taxon. 22(1): 17-41.

Rizvi, S.N.H. 1970. East Pakistan District Gazetteers for Sylhet. Government of East Pakistan Survices and General Administration Department, Dhaka. pp. 5-6.

Sarker, S.K. and Hossain, A.B.M.E. 2009. Pteridophytes of greater Mymensingh District of Bangladesh used as vegetables and medicines. Bangladesh J. Plant Taxon. 16(1): 47-56.

Siddiqui, K.U., Islam, M.A. Ahmed, Z.U. Begum, Z.N.T., Hassan, M.A., Khondker, M., Rahman, A. M., Kabir, S.M.H., Ahmad, M., Ahmed, A.T.A., Rahman, A.K.T. and \& Haque, E.U. (eds.) 2007. Encyclopedia of Flora and Fauna of Bangladesh, vol.-5, Asiatic Society of Bangladesh, Dhaka. pp. 1-391.

Singh, K.K., Saha, S. and Maheshwari, J.K. 1989. Ethnomedicinal uses of some ferns amongst the tribals of Uttar Pradesh. Indian Fern J. 6: 66-67.

Smitinand, T. and Larsen, K. (eds). 1975. Flora of Thailand, vol. 2, part 3, Applied Scientific Research Corporation of Thailand, Bangkok. pp. 1-280.

Smitinand, T. and Larsen, K. (eds). 1989. Flora of Thailand, Forest Herbarium, Royal Forest Department, Bangkok. Mem. Fac. Sci. Kyoto Univ. Biol. Sci. 3(4): 67-88.

Jain, S.K. and Rao, R.R. 1977. A handbook of field and herbarium methods. Today and Tomorrow's Printers and Publishers, New Delhi. pp. 1-157.

Tropicos. 2010. Missouri Botanical Garden. Missouri Botanical Garden, 4344 Shaw Boulevard, Saint Louis, Missouri 63110. http://www.tropicos.org.

Uddin, M.G. and Pasha, M.K. 1997. Systematic studies on the fern-allies of Bangladesh. Indian Fern J. 14: 23-32.

Uddin, M.G., Mirza, M.M. and Pasha, M.K. 1998. The medicinal uses of pteridophytes of Bangladesh. Bangladesh J. Plant Taxon. 5(2): 29-41.

Uddin, M.G. 2001. A taxonomic study on Leptosporangiate ferns of Bangladesh. M. Phil. Thesis, Department of Botany, University of Chittagong. pp. 1-239

Uddin, M.G., Rahman, M.M., Sarker, S.K. and Pasha, M.K. 2001. A systematic account of the pteridophytic flora of greater Mymensingh District of Bangladesh. Bangladesh J. Plant. Taxon. 8(1): 65-80.

Uddin, S.B., Rahman, M.A., Uddin, M.G. and Pasha, M.K. 2008. Ethno-botancial uses of Pteridophyte from Chittagong Hill Tracts of Bangladesh. Nepal Journal of Plant Science 2(1): 89-93.

Uddin, S.N. and Hassan, M.A. 2012. Pteridophyte Flora of Rampahar and Sitapahar Reserve Forests under Rangamati District in Bangladesh. Dhaka Univ. J. Biol. Sci. 21(2): 153-161.

Wu, C.Y., Raven, P.H. and Hong, D.Y. (Eds.) 1995-2013. Flora of China. Vols. 2-25. Science Press and Missouri Botanical Garden Press, Beijing and St. Louis. 